\title{
Where to draw the line: honor mindset increases retaliation in response to unfair behavior
}

\author{
Nic Flinkenflogel ${ }^{1} \cdot$ Sheida Novin ${ }^{2}$ (D) Anna van der Meulen ${ }^{1,3}$. \\ Lydia Krabbendam ${ }^{1}$ (D)
}

Accepted: 31 March 2020 / Published online: 11 April 2020

(c) The Author(s) 2020

\begin{abstract}
Past research has linked honor to a higher tendency for retaliation. A common method is to compare groups that are either low or high in honor. While effective, this does not account for within-group variation, nor isolate honor as a distinct construct from alternative differences between groups that might affect the outcome. In the current study we investigated honor as a cultural mindset, using priming methodology in both Dutch participants (who are typically low in honor endorsement) and Dutch-Turkish and Dutch-Moroccan participants (who are typically high in honor endorsement), while controlling for trait endorsement of honor values. To quantify retaliation, we used two social dilemmas: presenting an unequal offer in the Ultimatum Game, and chips being taken in the Justice Game. The results showed that priming honor resulted in an increased willingness to pay for punishment in the Justice Game, but not higher rejection in the Ultimatum Game. This suggests that unfairness by itself is not sufficient to trigger retaliation; rather, a pronounced transgression is required-in this case something being taken what is considered rightfully yours. Furthermore, decision-making in both social dilemmas was not associated with cultural background or honor endorsement. This indicates that an honor mindset needs to be salient to affect decision-making, and can affect behavior over and above the endorsement of honor values.
\end{abstract}

Keywords Honor $\cdot$ Priming $\cdot$ Retaliation $\cdot$ Cultural mindset $\cdot$ Decision-making

Sheida Novin

s.novin@uu.nl

1 Department of Clinical, Neuro- \& Developmental Psychology, VU University, Amsterdam, The Netherlands

2 Department of Developmental Psychology, Utrecht University, Utrecht, The Netherlands

3 Leiden Institute of Advanced Computer Science, Leiden University, Leiden, The Netherlands 


\section{Introduction}

Social norms, including being fair to others, are an essential part of human society such that they aid social interaction, conformity, and cooperation (Buckholtz and Marois 2012; Decety and Yoder 2017; Fehr and Fischbacher 2004). As such, enforcement of such norms is considered universal and is observed in children as young as three years (Cowell and Decety 2015; Jensen et al. 2014; Schmidt et al. 2012). People have the tendency to act upon social norm violations, but how they act greatly differs between individuals and societies (Prentice 2018). When treated unfairly, some may mildly protest while others may retaliate, even at great personal cost. Past research has linked honor to a higher tendency for retaliation, but this work mainly (1) focused on situations in which people are insulted/ humiliated, (2) self-reported emotional and behavioural responses to hypothetical scenarios rather than actual behavior, and (3) used a group-comparison approach that cannot directly test the downstream consequences of honor on retaliation. In the current study we move beyond existing studies by experimentally testing whether an activated honor mindset directly influences behavorial retaliation to unfair behavior.

In the literature, honor is considered a multifaceted construct that involves the self (personal honor), the social group (group honor), and gender roles and norms (i.e., masculine and feminine honor) (e.g. Cihangir 2013; Cohen et al.; Cross et al. 2014; Leung and Cohen 2011; Novin and Oyserman 2016). In this study we focus on personal honor, which on the one hand includes concerns for one's integrity such as being honest, trustworthy, and being true to one's principles (Cross et al. 2014; Peristiany 1965). On the other hand, personal honor also includes concerns for one's social image or reputation such as wanting to be respected and positively recognized by important others and defending oneself when disrespected or humiliated (Cross et al. 2014; Peristiany 1965). Which facet of personal honor is most salient and most highly endorsed, may differ across cultural groups (e.g., Uskul et al. 2012; Guerra et al. 2013; IJzerman et al. 2007). For example, in one study comparing Dutch and Turkish participants, Turkish participants reported higher endorsement of honor values related to integrity, while these groups did not differ in their endorsement of honor values related to social image (Novin et al. 2015). Despite these cultural group differences, honor values are considered to be understandable across cultures, including in so-called dignity or face cultures where honor is not salient on a daily basis or where these honor values are not highly endorsed (Cross et al. 2014; Guerra et al. 2013; IJzerman and Cohen 2011; Novin and Oyserman 2016).

The majority of research on personal honor focused on how people react to situations in which their social reputation is threatened, for example when insulted or humiliated. Typically, these studies compare groups that highly endorse honor values (high honor groups) from those that endorse honor values to a lesser extent (low honor groups). These high honor groups come from cultures of honor (e.g., the Mediterranean, the Middle East, Latin America, and Southern United States) and low honor groups typically come from dignity cultures (e.g., Western Europe, 
Northern United States) (Barnes et al. 2012; Cohen et al. 1996; Rodriguez Mosquera et al. 2008; Uskul et al. 2015; van Osch et al. 2013). These studies have shown that individuals from high honor groups tend to report more negative emotions (i.e. anger, shame, but not fear) that motivate to defend and protect their social reputation than those from low honor groups (IJzerman et al. 2007; Rodriguez Mosquera et al. 2000, 2002). Consequently, these individuals are also more likely to respond with either overt (e.g., physical aggression) or subtle (e.g., gossip) forms of retaliation when insulted or humiliated (Archer and Coyne 2005; Cohen et al. 1996; Stewart 1994; Uskul et al. 2015). A classical study by Cohen et al. (1996) demonstrated that men from the South of the U.S. (high honor group) were both more physiologically and psychologically prepared for aggression than their Northern counterparts (low honor group) after a confederate bumped into them, as measured by their higher levels of testosterone and cortisol, and greater willingness to engage in aggressive or dominant displays of behavior.

One prominent theory that helps to understand these differences in reactions postulates that cultures of honor develop in regions with low governmental involvement, lawlessness, and economic insecurity (Brown 2016; Cohen et al. 1996; Leung and Cohen 2011). In such insecure and resource-poor environments, it is the responsibility of the individual to be vigilant and respond to threats that could disrupt social order and damage reputation, as social chaos or loss of materials goods may threaten an individual's chance of survival (Nowak et al. 2016). Unpunished threats might signal that one cannot defend oneself or one's group, paving the way for more serious transgressions (Nisbett et al. 1996). As such, a person's honor relies on a mixture of attitudes that help to maintain a stable social context and a positive personal reputation, including doing the right thing, being positively evaluated by others, and intolerance of disrespect and transgressions to social norms.

The relationship between honor and retaliation beyond situations involving insults/humiliation has received much less attention. Moreover, retaliation in the form of actual behavioral punishment (i.e., taking action to punish the wrongdoer) is not measured (except for the descriptive literature on honor killings, e.g., Asquith 2015; Gill et al. 2014; Kulczycki and Windle 2011). To the best of our knowledge only one study addressed these drawbacks (Uskul et al. 2015). In this study Turkish (high honor group) and North American (low honor group) participants were asked to write an essay and were subsequently treated unfairly by being accused of violating a moral norm (i.e., being dishonest). The results revealed that Turkish participants were more likely to retaliate by assigning more difficult tasks and sensory tasks with a higher level of intensity to their attacker, compared to the American participants. The role of individuals' honor endorsement on retaliatory responses was unknown.

Another drawback of prior work concerns the group-comparison approach that is often used. While such approach serves to illustrate differences between cultural groups, it remains difficult to attribute the observed effects to honor specifically, rather than the aggregate of cultural differences between two regions (such as local practices, traditions, and religion). As detailed next, the current study is an attempt to address these drawbacks. 


\section{Current study}

Recently, the honor field has started to consider honor as a universally accessible mindset, which exists across societies, but differs in its prevalence (Cross et al. 2014; Novin and Oyserman 2016). This perspective implies that honor is more chronically activated in high honor societies such as Turkey based on situational constraints (Cross et al. 2014; Kardam, Alpar, Yuksel, and Ergun 2005), but that it can also be activated in low honor societies (e.g., dignity societies such as the Netherlands) by contextual cues (Flinkenflogel et al. 2017; Oyserman and Lee 2008). According to this perspective, honor can be considered a dynamic cultural mindset that contains specific content, goals, and procedures, which affect cognition and social behavior once activated. Moreover, this perspective opens the possibility to use an alternative approach to study honor. Namely, by using priming methodology researchers can activate an honor mindset, and investigate the downstream consequences on behavior. Indeed, previous research has shown that activating an honor mindset affects decision-making, regardless of an individual's endorsement of honor values (Novin and Oyserman 2016).

In the current study we used such an experimental approach to examine the influence of an activated honor mindset on retaliation when one is treated unfairly. Unfair behavior can be considered a transgression of a social norm (Camerer and Fehr 2004; Stallen et al. 2018; Weber et al. 2004). We operationalized retaliation as costly punishment behavior, and used two social dilemma paradigms that allowed us to measure the actual behavior. To test the influence of an activated honor mindset regardless of cultural background, we included a diverse group of participants from backgrounds that in the literature represent low honor and high honor groups. The low honor group consisted of ethnically Dutch participants who typically have a weak concern for honor values (Leung and Cohen 2011; Rodriguez Mosquera et al. 2000, 2002). The high honor group consisted of Turkish-Dutch and MoroccanDutch participants, who typically have a stronger concern for honor values (Cihangir 2013; Novin et al. 2015; Rodriguez Mosquera et al. 2008).

To prime honor, half of the participants completed an honor questionnaire before the social dilemma paradigm (honor prime condition), and the other half completed the same questionnaire after the social dilemma paradigm (control condition). This design provided the opportunity to prime an honor mindset, while simultaneously controlling for the effects of trait endorsement of honor values. As both aspects of personal honor (concern for one's integrity and concern for one's social image) are likely to be threatened when being treated unfairly, the honor questionnaire included statements from both facets of honor.

Where research assessing the relationship between honor and retaliation has typically used self-report questionnaires (Barnes et al. 2012), we used two social dilemmas to measure behavioral retaliation. While valuable, self-reports can be subjectively biased, as they do not provide a direct measure of behavior including associated costs and benefits (Adams et al. 1999; Van de Mortel 2008). Social dilemmas provide a means to quantify aspects of behavior in simplified representations of social interactions that pit benefit for self against benefit for others (Weber et al. 2004). Pedersen et al. (2014) used a dictator game to examine the relationships 
between honor value endorsement and emotions after being treated unfairly. In this study, participants were endowed with $\$ 5$, and would then have either $\$ 1$ or $\$ 4$ taken by a 'dictator', after which they completed several questionnaires. The authors found that participants who endorsed honor reported higher levels of anger and envy. However, this study did not measure behavioral response to examine how the experience of unfairness translates to action.

In the current study, we employed two social dilemmas that have been linked to being treated unfairly; the Ultimatum Game (UG) (Güth et al. 1982) and the Justice Game (JG) (Stallen et al. 2018). The UG is a two-player social dilemma where a Proposer makes an offer to split a certain sum, and the Responder can either accept or reject, with rejection resulting in forfeit of all possible gains for both players. In other words, the Responder can punish the Proposer by rejecting the offer at a personal cost. In the JG, two players are endowed with a sum, and a Taker can take a sum of chips from the Partner. However, the Partner can respond by sacrificing chips to retaliate, with each sacrificed chip decreasing the income of the Taker by three times. Both games have been demonstrated to elicit negative emotions in response to unfairness (Sanfey et al. 2003; Stallen et al. 2018), and measure a form of costly punishment; i.e., participants need to forfeit a share of their possible winnings in order to retaliate. However, previous research has shown that something being taken that can be viewed as rightfully yours (e.g. chips or money) is viewed as a greater transgression compared to receiving an unfair division (Ben-Shakhar et al. 2007; Korenok et al. 2014; Stallen et al. 2018). The difference between the games can therefore be viewed as a general indication of unfairness (UG), or more pronounced transgression (JG).

Despite these differences between the games, our expectations were that making an honor mindset salient would result in increased retaliation as seen by greater rejection rates in the $\mathrm{UG}$, as well as higher levels of punishment in the JG, in both the low honor and high honor groups. We further expected the honor prime to affect behavior over and above trait endorsement of honor. Finally, we expected the high honor group to overall display both higher rejection rates as well as higher levels of punishment compared to the low honor group.

\section{Method}

\section{Participants}

Due to the difficulty of collecting a representative sample on or near campus, which would reflect the desired variety in cultural and socio-economic background, participants were recruited via internet fora, student associations, and social networks. Inclusion criteria were having either a Dutch, Dutch-Turkish, or Dutch-Moroccan background and being fluent in Dutch, as the experiment was conducted in Dutch. Active informed consent was obtained, and experimental procedures were reviewed and found in accordance with the guidelines of the ethical committee of the Faculty of Behavioral and Movement Sciences. The total sample consisted of 226 participants (35\% male; 61\% Dutch, 23\% Turkish, 16\% Moroccan; $M_{\text {age }}=25.24$, 
$S D=4.92$, age range: $18-40$ years). The Dutch-Turkish and Dutch-Moroccan (high honor group, $n=89,42 \%$ male) participants were contrasted with the Dutch participants (low honor group, $n=137 ; 31 \%$ male) in analyses. Participants were randomly assigned to the two conditions (no-prime control condition: $n=108,31 \%$ male, $56 \%$ Dutch; honor prime condition: $n=118,39 \%$ male, $64 \%$ Dutch).

\section{Procedure and materials}

The study was conducted online via Qualtrics software and participants performed the experiment in their home environment. They were first provided with an explanation about the procedure, including instructions for the UG. They were informed the experiment would last twenty minutes, and that they could stop at any moment. Participants then provided consent before continuing with the experiment. In the priming condition, participants commenced with the honor questionnaire, which served as a prime. They proceeded with the one-shot UG, before being linked through to a separate website to participate in the JG, which was programmed in HTML. They received instructions for the JG, and played eight subsequent rounds. Upon completion, participants were linked back to Qualtrics where they filled out the control questions and demographics. The UG always preceded the JG, because the duration of the JG would require a repetition of the prime, whereas the one-shot UG was considered sufficiently short to be able to detect priming effects on the JG. In the noprime condition participants commenced directly with the UG followed by the JG, and subsequently filled out the honor questionnaire, as well as the control questions and demographics. The experiment concluded with a debriefing. Upon completion, participants were paid a base fee of $€ 2$, with an additional bonus up to $€ 3,50$ based on their choices in the games (i.e., whether they accepted the UG offer, and the average amount of chips left on four randomly selected trials in the JG; the total possible amount of 200 chips equaled $€ 2$, with each increment of 10 points rendering $€ 0.10$ ).

\section{Ultimatum game}

The first social dilemma was a one-shot UG. The dependent variable was rejection rate: participants decided whether to accept or reject an offer where the anonymous proposer would receive 7 coins, and the participant 3 . One coin translated to 50 eurocents, so the participant was making a choice to accept 1.50 euro. The offer was framed as being proposed by a participant who had previously participated in the experiment.

\section{Justice game}

The second social dilemma was the JG, which measures norm enforcement by means of punishment (Stallen et al. 2018). The JG is a two-player social dilemma in which one person is assigned the role of Taker, and the other that of Partner. At the beginning of each round each player receives an endowment of 200 chips. The Taker begins with the possibility of taking an amount of chips 
from the Partner, ranging from 0 to 100 in increments of 25. The Partner can then choose to sacrifice an amount of chips ranging from 0 to 100 (in increments of 10) to punish the Taker. Each chip spent on punishment decreases the income of the Taker by three chips. For example, if a Taker takes 50 chips from the Partner, the Partner keeps 150 chips, while the Taker now has 250 chips. If the Partner subsequently wants to punish the Taker by allocating 50 chips on punishment, the income of the Taker decreases by 150 chips $(50 \times 3)$. The Taker then has 100 chips left (250-150), as does the Partner (150-50). After a round is completed, a new round starts with a different Taker.

In the instruction, participants were informed they would be randomly assigned to either role, and if assigned the role of Partner, the Taker's choices were recorded responses from participants who had previously played the JG. However, participants were always assigned the role of Partner, and faced eight preprogrammed choices of the Taker in random order; specifically, four 'baseline' trials in which the Taker did not take any chips, and one trial each in which the Taker took either 25, 50, 75 or 100 chips.

To probe whether the participants had fully understood the instructions, we included two control questions at the end of the study to infer what they thought about playing against multiple opponents, and if they understood that the amount of chips they selected to punish the Taker were sacrificed, rather than added to their stack. When participants indicated they had not completely understood the JG based on these questions, they were excluded from analyses. In total, 21 participants were removed from the total sample for the JG analysis (11 from the low honor group, 10 from the high honor group). The remaining sample for the analysis of the JG consisted of a total of 205 participants (noprime control condition: $n=92,36 \%$ male, 55\% Dutch; $M_{\text {age }}=23.21, S D=3.06$, age range: $18-40$ years; honor prime condition: $n=113,41 \%$ male, $63 \%$ Dutch; $M_{\text {age }}=26.50, S D=4.63$, age range: $18-40$ years).

\section{Honor questionnaire}

We used an honor scale to make an honor mindset salient, similar to the procedures used by Novin and Oyserman (2016). The scale consisted of 11 items derived from the honor questionnaire used by IJzerman et al (2007). The original scale contains items about integrity, reputation, and family honor. To bring to mind personal honor rather than family honor, we omitted items on family honor in our scale. Example items reflecting honor integrity are "I maintain my honor when I live according my own values and principles" and "I maintain my honor when I'm honest to others". Example items reflecting social reputation are "To maintain my honor I should always be prepared to defend myself" and "I think that being humiliated in a public place would be one of the situations that would damage my honor most". Participants' endorsement of honor values was measured on a 7-point Likert scale $(1=d o$ not agree at all, $7=$ totally agree; $\alpha=0.77)$. A mean score of the items was calculated and included as trait honor in the analysis. 


\section{Analysis plan}

To analyze whether making an honor mindset salient affected the outcome of the UG, we conducted a $2 \times 2$ stepwise binary logistic regression with condition (control [-1] vs. honor [1]) and group (low-honor [-1] vs. high-honor [1]) as predictors in the first step to analyze the main effects, and included the condition by group interaction as predictor in the second step. The binary dependent variable was whether the participant accepted or rejected the offer. To examine whether the condition effects would hold over and above trait endorsement levels of honor values, the analysis was subsequently repeated including endorsement of honor values as a covariate.

To analyze whether the effects of a salient honor mindset affected the outcome of the JG, we conducted a $2 \times 2 \times 4$ repeated measures ANOVA with the between-subjects factors condition, group, and the condition by group interaction and the withinsubjects variable amount of chips taken $(0,25,50,75,100)$ as predictors. The four baseline trials where the Taker did not take any chips were averaged together for the analysis. The dependent variable was the amount of chips participants paid to punish the Taker (0-100), in increments of 10. Again, the analysis was repeated including honor endorsement as a covariate. The significance level was set at $\alpha \leq 0.05$ for all analyses.

\section{Results}

\section{Descriptives}

To examine differences in honor endorsement (range $=1.64-6.73, M=4.56$, $S D=0.80)$ as a function of group, we performed a univariate analysis. Participants in the high honor group did not differ in their reported honor endorsement $(M=4.72$, $S D=0.89)$ compared to those in the low honor group $(M=4.48, S D=0.72)$, $F(1,224)=3.320, p=0.070, d=0.24$. To examine differences in honor endorsement before and after the games, we performed a second univariate analysis as a function of condition. The results revealed that participants' honor endorsement in the honor prime condition, who completed the honor scale prior the games, did not differ from those in the no-prime control condition, who completed the honor scale after the games, $F(1,224)=0.548, p=0.460$.

\section{Ultimatum game}

To test the main effects of condition and group a logistic binary regression was performed with condition and group as categorical factors. The analysis revealed no significant differences between the honor prime and no-prime control condition, Wald $\chi^{2}(1,226)=0.005, p=0.944$, such that both conditions rejected the offer equally ( $55 \%$ and $56 \%$ respectively). Similarly, the low and high honor group did not 
differ in their rejection rates $\left(51 \%\right.$ and $63 \%$, respectively), Wald $\chi^{2}(1,226)=3.002$, $p=0.083$.

Next, the condition by group interaction effect was included in the second step of the model to analyze the differences between conditions within the low and high honor groups, as well as the differences between groups within the honor prime and no-prime control conditions. However, the analysis did not reveal significant differences in rejection rate between condition or group. In addition, the interaction between condition and group was not significant, Wald $\chi^{2}(1,226)=0.549$, $p=0.459$. Furthermore, there was no difference in rejection rate between the honor prime (52\%) and the no-prime control condition (49\%) in the low honor group, Wald $\chi^{2}(1,226)=0.161, p=0.688$, or between the honor prime $(59 \%)$ and the noprime control condition $(66 \%)$ in the high honor group, Wald $\chi^{2}(1,226)=0.393$, $p=0.531$. Similarly, the difference in rejection rate between the low (52\%) and high (59\%) honor group in the honor prime condition was not significant, Wald $\chi^{2}(1$, $226)=0.518, p=0.472$, nor was the difference between the low $(49 \%)$ and high $(66 \%)$ honor group in the no-prime control condition, Wald $\chi^{2}(1,226)=3.005$, $p=0.083$. Finally, adding trait honor endorsement as a covariate did it affect the outcome of the other factors on the UG, nor was it related to the outcome of the UG, Wald $\chi^{2}(1,226)=1.049, p=0.306$.

\section{Justice game}

The analysis revealed a significant main effect of condition, $F(1,201)=6.542$, $p=0.011, d=0.36$; participants in the honor prime condition punished more than those in the control condition (Fig. 1). There was no significant difference between the low and high honor group, $F(1,200)=1373, p=0.243$ (Fig. 2), nor a significant

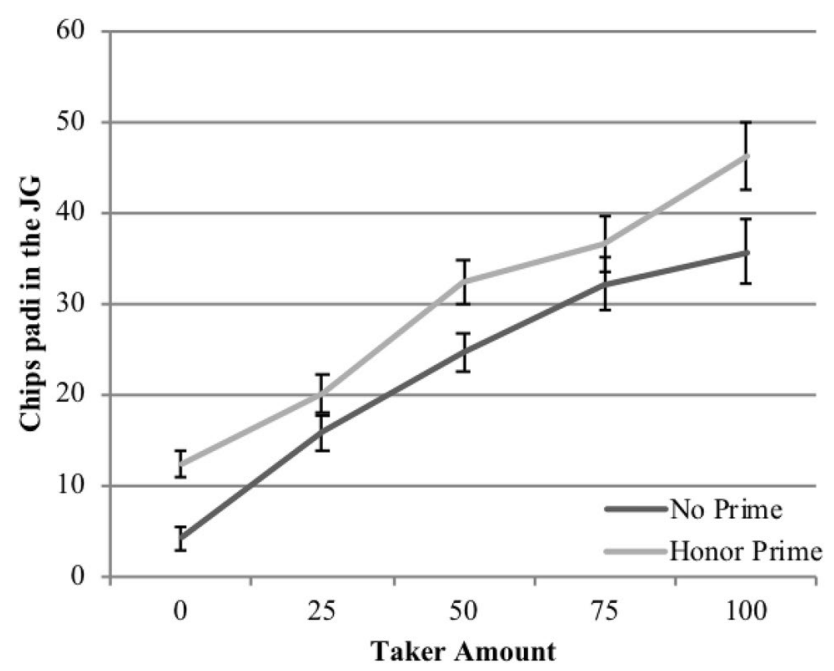

Fig. 1 Average rates participants in the control and honor prime condition paid to punish per amount of the Taker in the JG 


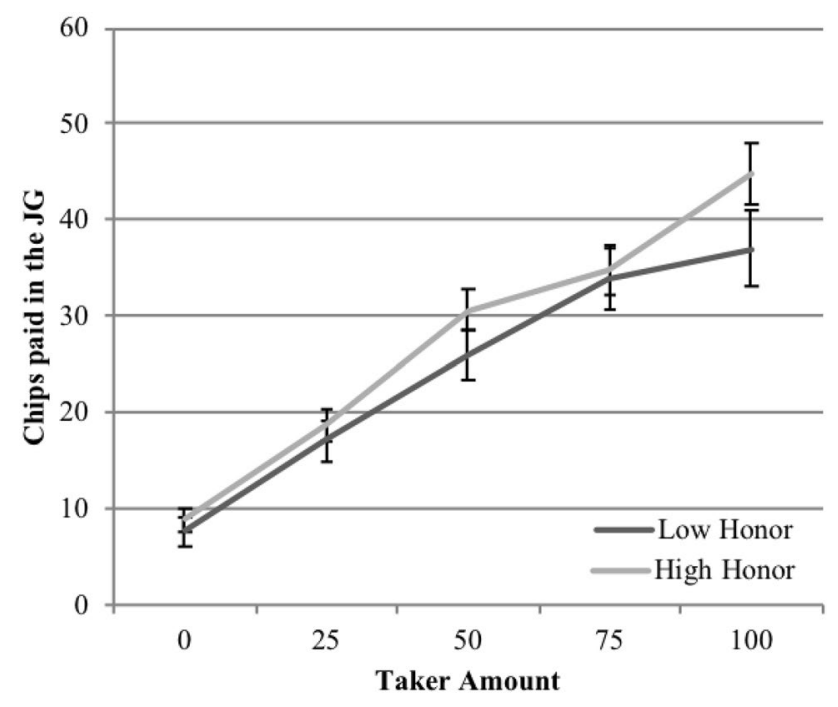

Fig. 2 Average rates participants in the low and high honor group paid to punish per amount of the Taker in the JG

interaction between condition and group, $F(1,200)=2.678, p=0.100$. Finally, participants punished more with higher amounts of the Taker, $F(4,804)=95.315$, $p<0.001, d=43.58$, but the amount taken did not interact with the other factors.

The subsequent ANCOVA analysis revealed that trait honor endorsement did not attenuate the main effect of condition, $F(1,200)=6.614, p=0.011, d=0.36$, nor did it correlate with punishment, $F(1,200)=0.321, p=0.571$, indicating that endorsement of honor values did not affect the outcome of the prime on punishment, nor was it associated with punishment level.

\section{Discussion}

Modern Western society is a mixture of different cultures, with each having their own standards for 'proper' behavior. What seems appropriate behavior in one culture, might easily seem inappropriate in a different culture (Leung and Cohen 2011). One of these examples is the culture of honor, in which it was imperative for survival to keep social order and defend one's reputation by taking matters into one's own hand and punish wrongdoers (Cohen et al. 1996). In the current research we operationalized honor as a dynamic culture mindset (Novin and Oyserman 2016), and used priming methodology to investigate how an activated honor mindset affected retaliation against social norms in the form of unfair behavior. We employed two social dilemmas that measure costly punishment in the form an actual behavior, but vary in their framing. The first social dilemma was the UG, which consisted of a marginally unfair offer that could either be accepted or rejected. The second dilemma was the JG, which measured the tendency to punish after chips were taken 
away. Contrary to our expectations, the findings indicated that making an honor mindset salient did not result in higher rejection rates in the $\mathrm{UG}$ - but did result in a higher willingness to pay for punishment in the JG, over and above endorsement levels of honor values. Furthermore, neither cultural background nor honor endorsement was associated with punishment, nor were these factors associated with the effect of the mindset prime.

The distinct effects in the UG versus the JG were unexpected, but a possible explanation might be due to the different framing of the dilemmas. The UG offer can be viewed as a mild social transgression; while the participants were offered a marginal share of a sum that can be labeled as 'unfair', every offer above the minimum can equally be viewed as a courtesy, and accepting entails receiving more than they had before. In the JG however, the Taker removed chips that were initially considered to belong to the participant. Therefore, the act of removing chips can be considered a greater violation than an unfair proposal (Stallen et al. 2018). An activated honor mindset includes the concern for sticking to your principles, defending what is yours, receiving and deserving respect from others, and acting against moral violations. Individuals with an activated honor mindset draw the line in the JG, displaying more willingness to pay to punish the other for unfair behavior, compared to those without an activated honor mindset. In other words, punishment does not simply occur in response to unfairness, i.e., materialized in resource inequality resulting from social comparison (receiving less than someone else), but is confined to a perceived personal transgression (e.g. something being taken that is considered rightfully yours).

This reasoning is in line with previous research suggesting that people who highly endorse honor values do not necessarily have a general conflict-oriented attitude, but might display a greater tendency to engage in conflict once a threshold is passed. For instance, Shafa et al. (2015) found that while people who endorse honor values showed more aggression during conflict, they were more accommodating towards others in their initial approach prior to conflict. In a similar vein, it is possible that while the proposed UG offer is an uneven distribution, it may not necessarily be perceived as a transgression. The act of removing one's chips in the JG on the other hand is.

Another possibility for the different findings in the UG and the JG might be due to the experimental design. The JG always preceded the UG and the unequal offer in the UG game itself might have served as an honor prime. That is, the unequal offer might have been perceived as an honor threat. As a result, it could have been that an honor mindset was more readily accessible or more strongly prevalent in the experimental honor mindset condition during the JG than the UG, causing stronger effects in the JG.

In addition, we found that trait endorsement of honor did not correlate with costly punishment, nor did it affect the effect of the prime. This aligns with previous findings by Novin and Oyserman (2016), who also reported the effect of a similar honor prime on decision-making, independent of honor endorsement. The authors suggest that regardless of whether individuals explicitly endorse honor values, an honor mindset can be brought to mind by subtle environmental cues, which subsequently influence implicitly how people think and act (Novin and Oyserman 2016). Our 
findings suggest that endorsing honor values is not sufficient to increase punishment in a social dilemma like the JG, and that it is necessary to have honor on the mind in that situation.

Nevertheless, these insights are relevant to understand the bicultural TurkishDutch and Moroccan-groups within the Dutch society. In this study the TurkishDutch and Moroccan-Dutch participants reported somewhat higher levels of honor endorsement than the Dutch participants, but this difference was not significant, probably due to the student samples. Yet, the two cultures (e.g., Turkish and Dutch) that are incorporated in individuals with these bicultural backgrounds differ strongly with regard to their emphasis on honor (Merz et al. 2009; Stupar et al. 2014). It is therefore likely that many individuals with a Turkish-Dutch and Moroccan-Dutch background encounter situations in which an honor mindset is activated on a daily basis (for instance when they are with their (extended) family) as well as situations where this is not the case (for instance at work or school). Interestingly, the current results suggest that behavior such as retaliation becomes more likely in situations where an honor mindset is made salient but not in other contexts. This suggests that these bicultural individuals can and might switch between mindsets depending on the immediate situation (Hong et al. 2000).

\section{Limitations and future recommendations}

Our study is the first to study how an honor mindset influences retaliation to being treated unfairly. Yet as any study, our study has a set of limitations that could be taken into account in future work. One limitation of the study is that participants performed the study online in their own rather than a laboratory environment. While this was the most efficient way to recruit participants, there is no way to control whether participants were distracted while engaging in the task, or what was on their mind during the no-prime control condition. Especially participants in the high honor group could have been affected by situational cues in their home environment which are more likely to trigger an honor mindset. However, if this were the case this would likely have led to a between-group difference in the control condition, and cannot account for the observed main effect of the prime (in the JG). Furthermore, there is a case to make that the opposite is equally true; an experiment performed at the university laboratory is likely to trigger an individualistic mindset.

Second, we included a group of bicultural individuals (i.e. Turkish-Dutch, Moroccan-Dutch), rather than a monocultural group recruited in the countries of origin. Even though second and third generation bicultural individuals still adhere to honor values, their behavior in society may to varying extents be influenced by the individualistic culture of the country they currently live in Merz et al. (2009). It is possible that this has reduced group differences in the outcome of the social dilemmas. Future research could consider measuring retaliation in the form of costly punishment by comparing two culturally distinct groups (e.g., those from a dignity vs. honor culture), while making corresponding mindsets (individualistic vs. honor mindset, respectively) salient. 
Furthermore, it should be noted that the two dilemmas differed in more aspects than degree of unfairness. First, the UG is a one-shot dilemma, whereas the JG involves multiple rounds with different Takers. This could have rendered the JG more sensitive to differences between the conditions. Second, the participant has a more agentic role in the JG as he or she can tailor the level of punishment, whereas the UG only allows for a relatively passive response.

Finally, while the majority of research on honor has suggested that people from typical honor cultures tend to display a higher tendency for retaliation, they can equally be more cooperative and friendly (Beersma et al. 2003; Harinck et al. 2013). A recommendation for future research would therefore be to include a social dilemma paradigm that measures cooperation such as the public goods game, to test whether an active honor mindset encompasses the broad scope of 'proper behavior'.

\section{Conclusion}

In the current study we used priming methodology to make an honor mindset salient, and examined its effects on retaliation in two social dilemmas. While an unfair offer in the UG was not sufficient to increase costly punishment in the form of rejection, making an honor mindset salient did result in a higher willingness to pay for punishment following clear transgressions in the JG. This effect was present independent of trait endorsement of honor values, and between-group cultural differences, which did not correlate with punishment behavior. The results support the notion that honor is a universally accessible mindset, that contains a distinct set of rules, motivations, and cognition, and accordingly, can affect the tendency for retaliation when made salient based on contextual cues-regardless of one's cultural orientation and background.

Author contributions NF, LK, SN and AM conceptualized the study, NF, SN and LK wrote the article, $\mathrm{NF}$ and AM collected and analyzed the data. All authors have given final approval for publication.

Funding This work was funded by a VICI grant from the Netherlands Organization for Scientific Research (453011-005, Krabbendam).

\section{Compliance with Ethical Standards}

Conflicts of interest The authors declare that they have no conflict of interest.

Ethical approval All procedures performed in studies involving human participants were in accordance with the ethical standards of the institutional research committee and with the 1964 Helsinki Declaration and its later amendments or comparable ethical standards.

Informed consent Informed consent was obtained from all participants. 
Open Access This article is licensed under a Creative Commons Attribution 4.0 International License, which permits use, sharing, adaptation, distribution and reproduction in any medium or format, as long as you give appropriate credit to the original author(s) and the source, provide a link to the Creative Commons licence, and indicate if changes were made. The images or other third party material in this article are included in the article's Creative Commons licence, unless indicated otherwise in a credit line to the material. If material is not included in the article's Creative Commons licence and your intended use is not permitted by statutory regulation or exceeds the permitted use, you will need to obtain permission directly from the copyright holder. To view a copy of this licence, visit http://creativecommons.org/licen ses/by/4.0/.

\section{References}

Adams, A. S., Soumerai, S. B., Lomas, J., \& Ross-Degnan, D. (1999). Evidence of self-report bias in assessing adherence to guidelines. International Journal for Quality in Health Care, 11(3), 187-192.

Archer, J., \& Coyne, S. M. (2005). An integrated review of indirect, relational, and social aggression. Personality and Social Psychology Review, 9, 212-230.

Asquith, N. L. (2015). Honour, violence and heteronormativity. International Journal for Crime, Justice and Social Democracy, 4(3), 73-84.

Barnes, C. D., Brown, R. P., \& Osterman, L. L. (2012). Don't tread on me: Masculine honor ideology in the US and militant responses to terrorism. Personality and Social Psychology Bulletin, 38(8), 1018-1029.

Beersma, B., Harinck, F., \& Gerts, M. J. (2003). Bound in honor: How honor values and insults affect the experience and management of conflicts. International Journal of Conflict Management, 14(2), 75-94.

Ben-Shakhar, G., Bornstein, G., Hopfensitz, A., \& van Winden, F. (2007). Reciprocity and emotions in bargaining using physiological and self-report measures. Journal of Economic Psychology, 28(3), 314-323.

Brown, R. P. (2016). Honor bound: How a cultural ideal has shaped the American psyche. Oxford: Oxford University Press.

Buckholtz, J. W., \& Marois, R. (2012). The roots of modern justice: Cognitive and neural foundations of social norms and their enforcement. Nature Neuroscience, 15(5), 655-661.

Camerer, C. F., \& Fehr, E. (2004). Measuring social norms using experimental games: A guide for social scientists. In J. Henrich, R. Boyd, S. Bowles, C. F. Camerer, E. Fehr, \& H. Gintis (Eds.), Foundations of human sociality: Experimental and ethnographic evidence from 15 small-scale societies. New York: Oxford University Press.

Cihangir, S. (2013). Gender specific honor codes and cultural change. Group Processes \& Intergroup Relations, 16(3), 319-333.

Cohen, D., Nisbett, R. E., Bowdle, B. F., \& Schwarz, N. (1996). Insult, aggression, and the southern culture of honor: An "experimental ethnography.". Journal of Personality and Social Psychology, 70(5), 945-960.

Cowell, J. M., \& Decety, J. (2015). Precursors to morality in development as a complex interplay between neural, socioenvironmental, and behavioral facets. Proceedings of the National Academy of Sciences of the United States of America, 112(41), 12657-12662.

Cross, S. E., Uskul, A. K., Gerçek-Swing, B., Sunbay, Z., Alözkan, C., Günsoy, C., et al. (2014). Cultural prototypes and dimensions of honor. Personality and Social Psychology Bulletin, 40(2), 232-249.

Decety, J., \& Yoder, K. J. (2017). The emerging social neuroscience of justice motivation. Trends in Cognitive Sciences, 21(1), 6-14.

Fehr, E., \& Fischbacher, U. (2004). Social norms and human cooperation. Trends in Cognitive Sciences, $8(4), 185-190$.

Flinkenflogel, N., Novin, S., Huizinga, M., \& Krabbendam, L. (2017). Gender moderates the influence of self-construal priming on fairness considerations. Frontiers in Psychology, 8, 503.

Gill, A. K., Strange, C., \& Roberts, K. (2014). Honour killing and violence. LondonLondon: Palgrave Macmillan. 
Guerra, V. M., Giner-Sorolla, R., \& Vasiljevic, M. (2013). The importance of honor concerns across eight countries. Group Processes \& Intergroup Relations, 16(3), 298-318.

Güth, W., Schmittberger, R., \& Schwarze, B. (1982). An experimental analysis of ultimatum bargaining. Journal of Economic Behavior \& Organization, 3(4), 367-388.

Harinck, F., Shafa, S., Ellemers, N., \& Beersma, B. (2013). The good news about honor culture: The preference for cooperative conflict management in the absence of insults. Negotiation and Conflict Management Research, 6(2), 67-78.

Hong, Y.-Y., Morris, M. W., Chiu, C.-Y., \& Benet-Martinez, V. (2000). Multicultural minds: A dynamic constructivist approach to culture and cognition. American psychologist, 55(7), 709-720.

IJzerman, H., \& Cohen, D. (2011). Grounding cultural syndromes: Body comportment and values in honor and dignity cultures. European Journal of Social Psychology, 41(4), 456-467.

IJzerman, H., van Dijk, W. W., \& Gallucci, M. (2007). A bumpy train ride: A field experiment on insult, honor, and emotional reactions. Emotion, 7(4), 869-875.

Jensen, K., Vaish, A., \& Schmidt, M. F. (2014). The emergence of human prosociality: Aligning with others through feelings, concerns, and norms. Frontiers in Psychology, 5, 822.

Kardam, F., Alpar, Z., Yuksel, I., \& Ergun, E. (2005). The dynamics of honor killings in Turkey: Prospects for action. Ankara: UN Development Program and UN Population Fund.

Korenok, O., Millner, E. L., \& Razzolini, L. (2014). Taking, giving, and impure altruism in dictator games. Experimental Economics, 17(3), 488-500.

Kulczycki, A., \& Windle, S. (2011). Honor killings in the Middle East and North Africa: A systematic review of the literature. Violence against women, 17(11), 1442-1464.

Leung, A. K.-Y., \& Cohen, D. (2011). Within-and between-culture variation: Individual differences and the cultural logics of honor, face, and dignity cultures. Journal of Personality and Social Psychology, 100(3), 507-526.

Merz, E.-M., Özeke-Kocabas, E., Oort, F. J., \& Schuengel, C. (2009). Intergenerational family solidarity: Value differences between immigrant groups and generations. Journal of Family Psychology, 23(3), 291-300.

Nisbett, D., \& Cohen, D. (1996). Culture of honor: The psychology of violence in the South. Boulder, CO: Westview Press.

Novin, S., \& Oyserman, D. (2016). Honor as cultural mindset: Activated honor mindset affects subsequent judgment and attention in mindset-congruent ways. Frontiers in Psychology, 7, 1921.

Novin, S., Tatar, B., \& Krabbendam, L. (2015). Honor and I: Differential relationships between honor and self-esteem in three cultural groups. Personality and Individual Differences, 86, 161-163.

Nowak, A., Gelfand, M. J., Borkowski, W., Cohen, D., \& Hernandez, I. (2016). The evolutionary basis of honor cultures. Psychological Science, 27(1), 12-24.

Oyserman, D., \& Lee, S. W. (2008). Does culture influence what and how we think? Effects of priming individualism and collectivism. Psychological Bulletin, 134(2), 311-342.

Pedersen, E. J., Forster, D. E., \& McCullough, M. E. (2014). Life history, code of honor, and emotional responses to inequality in an economic game. Emotion, 14(5), 920-929.

Peristiany, J. G. (1965). Honour and shame: The values of Mediterranean society. London: Weidenfeld.

Prentice, D. A. (2018). Intervening to change social norms: When does it work? Social Research: An International Quarterly, 85(1), 115-139.

Rodriguez Mosquera, P. M. (2013). In the name of honor: On virtue, reputation and violence. Group Processes \& Intergroup Relations, 16(3), 271-278.

Rodriguez Mosquera, P. M., Fischer, A. H., Manstead, A. S. R., \& Zaalberg, R. (2008). Attack, disapproval, or withdrawal? The role of honour in anger and shame responses to being insulted. Cognition and Emotion, 22(8), 1471-1498.

Rodriguez Mosquera, P. M., Manstead, A. S., \& Fischer, A. H. (2000). The role of honor-related values in the elicitation, experience, and communication of pride, shame, and anger: Spain and the Netherlands compared. Personality and Social Psychology Bulletin, 26(7), 833-844.

Rodriguez Mosquera, P. M., Manstead, A. S. R., \& Fischer, A. H. (2002). The role of honour concerns in emotional reactions to offences. Cognition and Emotion, 16(1), 143-163.

Sanfey, A. G., Rilling, J. K., Aronson, J. A., Nystrom, L. E., \& Cohen, J. D. (2003). The neural basis of economic decision-making in the ultimatum game. Science, 300(5626), 1755-1758.

Shafa, S., Harinck, F., Ellemers, N., \& Beersma, B. (2015). Regulating honor in the face of insults. International Journal of Intercultural Relations, 47, 158-174.

Schmidt, M. F. H., Rakoczy, H., \& Tomasello, M. (2012). Young children enforce social norms selectively depending on the violator's group affiliation. Cognition, 124(3), 325-333. 
Stallen, M., Rossi, F., Heijne, A., Smidts, A., De Dreu, C. K., \& Sanfey, A. G. (2018). neurobiological mechanisms of responding to injustice. Journal of Neuroscience, 38(12), 2944-2954.

Stewart, F. H. (1994). Honor. Chicago: Chicago University Press.

Stupar, S., van de Vijver, F. J., Te Lindert, A., \& Fontaine, J. R. (2014). Multicultural attitudes mediate the relation between personality and perceived ethnic outgroup distance in the Netherlands. International Journal of Intercultural Relations, 38, 24-35.

Uskul, A. K., Cross, S. E., Günsoy, C., Gerçek-Swing, B., Alözkan, C., \& Ataca, B. (2015). A price to pay: Turkish and Northern American retaliation for threats to personal and family honor. Aggressive behavior, 41(6), 594-607.

Uskul, A. K., Cross, S. E., Sunbay, Z., Gercek-Swing, B., \& Ataca, B. (2012). Honor bound: The cultural construction of honor in Turkey and the Northern United States. Journal of Cross-Cultural Psychology, 43(7), 1131-1151.

Van de Mortel, T. F. (2008). Faking it: Social desirability response bias in self-report research. Australian Journal of Advanced Nursing, 25(4), 40-48.

van Osch, Y., Breugelmans, S. M., Zeelenberg, M., \& Bölük, P. (2013). A different kind of honor culture: Family honor and aggression in Turks. Group Processes \& Intergroup Relations, 16(3), 334-344.

Weber, J. M., Kopelman, S., \& Messick, D. M. (2004). A conceptual review of decision making in social dilemmas: Applying a logic of appropriateness. Personality and Social Psychology Review, 8(3), 281-307.

Publisher's Note Springer Nature remains neutral with regard to jurisdictional claims in published maps and institutional affiliations. 